Service social

\title{
Pratiques d'action communautaire en CLSC : acquis et défis d'aujourd'hui, sous la direction de Louis Favreau, René Lachapelle et Lucie Chagnon, Sainte-Foy, Presses de l’Université du Québec, 1993, 234 pages.
}

\section{Denis Plamondon}

Volume 43, numéro 2, 1994

Enfants, parents, intervenants

URI : https://id.erudit.org/iderudit/706662ar

DOI : https://doi.org/10.7202/706662ar

Aller au sommaire du numéro

Éditeur(s)

École de service social de l'Université Laval

ISSN

1708-1734 (numérique)

Découvrir la revue

Citer ce compte rendu

Plamondon, D. (1994). Compte rendu de [Pratiques d'action communautaire en CLSC : acquis et défis d'aujourd'hui, sous la direction de Louis Favreau, René Lachapelle et Lucie Chagnon, Sainte-Foy, Presses de l'Université du Québec, 1993, 234 pages.] Service social, 43(2), 159-161. https://doi.org/10.7202/706662ar d'utilisation que vous pouvez consulter en ligne. 


\section{RECENSIONS}

\section{PRATIQUES D'ACTION COMMUNAUTAIRE EN CLSC: ACQUIS ET DÉFIS D'AUJOURD'HUI}

Sous la direction de Louis Favreau, René Lachapelle et Lucie Chagnon Sainte-Foy, Presses de l'Université du Québec, 1994, 234 pages.

Le Regroupement québécois des intervenants et intervenantes en action communautaire en CLSC (RQIIAC) tenait son troisième colloque sur le thème Acquis et défis d'aujourd'hui, à l'Université du Québec à Hull en juin 1992. Pendant trois jours, 275 personnes, des praticiens et praticiennes de l'organisation communautaire en CLSC, s'y sont retrouvées.

Les auteurs disposaient, en plus des textes des vingt-cinq conférenciers, d'une vingtaine de rapports d'ateliers et d'une trentaine d'expériences d'organisation communautaire menées dans divers coins du Québec. Faisant suite à ce colloque, les trois auteurs, qui faisaient aussi partie du comité organisateur, ont choisi de rendre compte de l'évolution de ce réseau d'intervenantes et intervenants communautaires de CLSC à ce moment-ci de leur histoire plutôt que de faire le récit de la rencontre.

Le livre est structuré en cinq parties. La première partie comprend I'ensemble des textes rattachés directement au thème du colloque. On y retrouve toutes les interventions de la séance d'ouverture, à l'exception d'un compte rendu d'ateliers qui s'est tenu plus tard dans le colloque. Regroupés sous le titre de Pratiques d'action communautaire: citoyenneté et démocratie, les textes de cette section s'intéressent à l'action communautaire en CLSC en la situant au-delà d'une simple activité professionnelle, dans un horizon plus large avec une emprise sur le devenir même du Québec.

Les trois sections suivantes émanent de la réduction d'une dizaine de thématiques secondaires. Les auteurs ont regroupé les textes autour de trois dimensions de l'action communautaire en CLSC qu'ils estiment constitutives et complémentaires. La première dimension a trait à la réalité professionnelle de l'intervention collective dans un cadre institutionnel tel qu'un CLSC. La seconde correspond à l'appartenance des CLSC à un 
réseau public, aux divers enjeux liés à la récente réforme de la santé et des services sociaux. Quant à la troisième dimension, elle renvoie à l'articulation d'une intervention en fonction du développement des communautés locales et du soutien à des groupes sociaux.

La dernière section, Un réservoir d'expertise, fait la liste des expériences qui ont fait l'objet d'une présentation et donne les coordonnées des personnes qui les ont rapportées. Cette dernière partie comporte aussi une analyse du contenu du bulletin de liaison du RQIIAC au cours des sept premières années de publication. Le lecteur peut y trouver des informations sur les expériences présentées comme sur d'autres expériences.

Les travailleuses et travailleurs sociaux plus orientés vers I'intervention individuelle, mais ouverts à une autre manière $d^{\prime}$ intervenir, les futures intervenantes qui s'interrogent sur la méthodologie de l'intervention collective ainsi que les directions de CLSC qui veulent avoir I'heure juste sur I'intervention collective en CLSC trouveront leur profit à la lecture de cet ouvrage.

La première partie leur permet de saisir les fondements de I'organisation communautaire, ses modèles de référence, son développement et son influence sur le devenir historique du Québec. Le texte d'une trentaine de pages du professeur américain Harry C. Boyte, communicateur de prestige du colloque, est sans aucun doute aussi une pièce maîtresse de cette section et même de l'ouvrage. Tout en nous faisant connaître l'expérience américaine d'engagement des citoyens en action communautaire, Harry C. Boyte nous éveille aux risques du professionnalisme à outrance (tant dans les services publics que dans les organisations communautaires) qui réduit les citoyens à des simples clients, consommateurs de service. Ses propos rafraîchissants ouvrent des perspectives dynamisantes sur la politique, la participation active des citoyens, la vie privée et la vie publique, etc.

Les trois parties suivantes permettent de saisir à ce moment-ci de I'histoire les conditions mêmes de la pratique de l'organisation communautaire, particulièrement en CLSC : I'autonomie professionnelle, le partenariat CLSC - organismes communautaires, les corporations de développement communautaire, le monde rural, l'action communautaire et le politique, l'intervention communautaire et le mouvement des femmes ainsi que l'intervention communautaire et le décrochage en milieu scolaire. La cinquième section, Un réservoir d'expertise, aurait pu être traitée autrement. Les deux chapitres qui composent cette section, bien qu'ils contiennent des informations intéressantes, auraient pu se retrouver davantage en annexe, étant donné leur facture (listes, nomenclatures).

Mis à part l'intérêt pour les participants eux-mêmes à disposer des actes du colloque, les organisateurs et organisatrices communautaires tout comme les étudiantes qui se spécialisent dans ce mode d'intervention préféreront les deux autres ouvrages publiés précédemment dans la même 
collection. Sur l'organisation communautaire en général, on se référera davantage à Théorie et pratiques en organisation communautaire de L. Doucet et L. Favreau (1991). Sur I'organisation communautaire en CLSC, on voudra consulter CLSC et communautés locales. La contribution de l'organisation communautaire, de L. Favreau et Y. Hurtubise. Le fait que le livre Pratiques d'action communautaire en CLSC : acquis et défis d'aujourd'hui soit diffusé deux ans plus tard entraîne pour conséquence le développement et la diffusion de mêmes thèmes dans les deux ouvrages cités précédemment. C'est une contrainte insurmontable quand on veut éditer les produits d'un colloque.

En somme, un livre abordable tant dans sa teneur que dans son prix pour le lecteur qui veut se familiariser avec l'organisation communautaire, particulièrement celle qui se pratique en CLSC.

Denis PLAMONDON

Département des sciences humaines Université du Québec à Chicoutimi

\section{L'INCESTE ET LE RAPPORT À LA LOI}

Pierre Fortin, Pierre-Paul Parent, Donald Plante et Jean-Yves Thériault Rimouski, Groupe de recherche ETHOS, Université du Québec à Rimouski, 1988, 47 pages.

Cette courte monographie, qui s'inscrit dans le cadre des publications du groupe interdisciplinaire de recherche sur les enjeux éthiques des interventions professionnelles et sociales (Éthos), rend compte de la réflexion de trois auteurs sur les rapports à la Loi de l'inceste. L'analyse ne se réfère pas aux lois ou aux institutions, mais à la Loi «en tant que fonction qui régule les conditions et les exigences fondamentales de la rencontre de I'autre» (p. 6). À partir de l'étude de 214 documents publiés entre 1977 et 1987 dans des journaux et des revues populaires, les auteurs proposent trois lectures éthiques des textes traitant de l'inceste.

Dans le premier chapitre, Pierre Fortin dégage de la littérature sur I'inceste trois enjeux moraux associés à ce problème: I'enjeu de I'enfermement lié à l'isolement des personnes et au silence qui entoure ce phénomène, l'enjeu de mort qui découle du fait que l'inceste anéantit les possibilités d'épanouissement de l'enfant et l'enjeu de mensonge qui correspond, d'une part, à l'abus de confiance dont est victime l'enfant et, $\mathrm{d}^{\prime}$ autre part, à la confusion dans les rôles au sein de la famille.

Dans le deuxième texte, intitulé «Observations d'un lecteur d'articles non scientifiques sur l'inceste», Jean-Yves Thériault regroupe ses 